\title{
Topology and bistability in liquid crystal devices
}

\author{
A. Majumdar, ${ }^{1,2, *}$ C. J. P. Newton, ${ }^{2, \dagger}$ J. M. Robbins, ${ }^{1, \ddagger}$ and M. Zyskin ${ }^{1, \S}$ \\ ${ }^{1}$ School of Mathematics, University of Bristol, University Walk, Bristol BS8 1TW, United Kingdom \\ ${ }^{2}$ Hewlett-Packard Laboratories, Filton Road, Stoke Gifford, Bristol BS12 6QZ, United Kingdom \\ (Received 7 November 2006; revised manuscript received 2 March 2007; published 16 May 2007)
}

\begin{abstract}
We study nematic liquid crystal configurations in a prototype bistable device-the post aligned bistable nematic $(\mathrm{PABN})$ cell. Working within the Oseen-Frank continuum model, we describe the liquid crystal configuration by a unit-vector field $\mathbf{n}$, in a model version of the PABN cell. First, we identify four distinct topologies in this geometry. We explicitly construct trial configurations with these topologies which are used as initial conditions for a numerical solver, based on the finite-element method. The morphologies and energetics of the corresponding numerical solutions qualitatively agree with experimental observations and suggest a topological mechanism for bistability in the PABN cell geometry.
\end{abstract}

DOI: 10.1103/PhysRevE.75.051703

PACS number(s): 61.30.Jf, 11.10.Lm, 61.30.Dk, 61.30.Hn

\section{INTRODUCTION}

Liquid crystals are an intermediate phase of matter between the solid and liquid states. In the simplest liquid crystal phase, the nematic phase, the constituent rodlike molecules tend to align along a locally preferred direction. This mean direction of molecular alignment is described by a director field $\mathbf{n}(\mathbf{r})$, which is an unoriented unit-vector field so that the sign of $\mathbf{n}$ has no physical significance [1].

The existence of a locally preferred direction and the resulting anisotropic optical properties make liquid crystals very suitable for display devices. Most of the liquid crystal displays (LCDs) in use today, such as the twisted nematic and the super twisted nematic, are monostable [2]. They can support two optically contrasting states, only one of which is stable without an applied field. Recently, there has been considerable interest in developing bistable display technologies, where there are two or more stable, optically contrasting states $[3,4]$. Here, power is needed only to switch between the different states but not to maintain them.

Bistable nematic LCDs typically use a combination of complex surface morphologies and surface treatments to stabilize the different states [3-5]. This paper focuses on one such bistable device - the post aligned bistable nematic (PABN) device [4]. The PABN device consists of a liquid crystal layer sandwiched between two substrates. The lower substrate is featured by an array of microscopic posts, as shown in Fig. 1. The boundary conditions are a mixture of tangent and normal conditions in various parts of the geometry (referred to as homogeneous and homeotropic, respectively, in liquid crystal literature [1]). Tangent boundary conditions on a surface constrain the director $\mathbf{n}$ to be in the plane of the surface whereas normal boundary conditions constrain $\mathbf{n}$ to be perpendicular to the surface.

For a range of post heights, the PABN device is experimentally observed to be bistable [4]. It supports two opti-

\footnotetext{
*Electronic address: a.majumdar@bristol.ac.uk

†Electronic address: chris.newton@hp.com

‡Electronic address: j.robbins@bristol.ac.uk

${ }^{\S}$ Electronic address: m.zyskin@bristol.ac.uk
}

cally contrasting states with long-term stability - one bright and the other dark when viewed between crossed polarizers. Optical modeling suggests that the dark state corresponds to a liquid crystal configuration that tilts strongly around the microscopic posts whereas the bright state corresponds to a suppressed tilt profile around the posts, i.e., a comparatively planar configuration [4]. The high-tilt configuration is referred to as the tilted state and the low-tilt configuration as the planar state in the remainder of the paper.

We use topological arguments to study static director configurations in the PABN geometry. The admissible configurations in this geometry can be partitioned into distinct topological classes (for details see [6,7]) and one might expect a stable configuration (or local energy minimizer) for every such class. The key point is that topologically distinct stable configurations cannot be continuously deformed into each other and switching proceeds via creation or annihilation of topological defects and boundary condition violation $[5,8]$. Therefore, such topologically distinct stable configurations, if they exist and have similar free energies, lead to bistability in prototype devices.

Our study is related to basic questions about the existence and properties of multiple equilibrium configurations in complex geometries. For polyhedral geometries (such as a rectangular prism), we have addressed several general questions

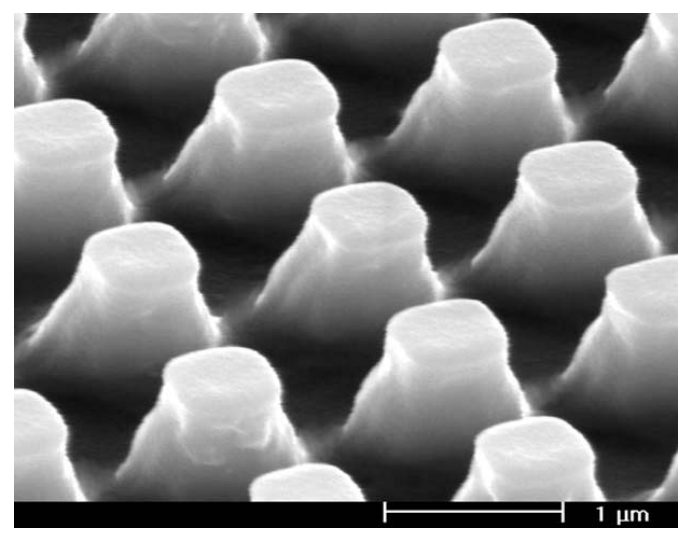

FIG. 1. The PABN device [4]. 


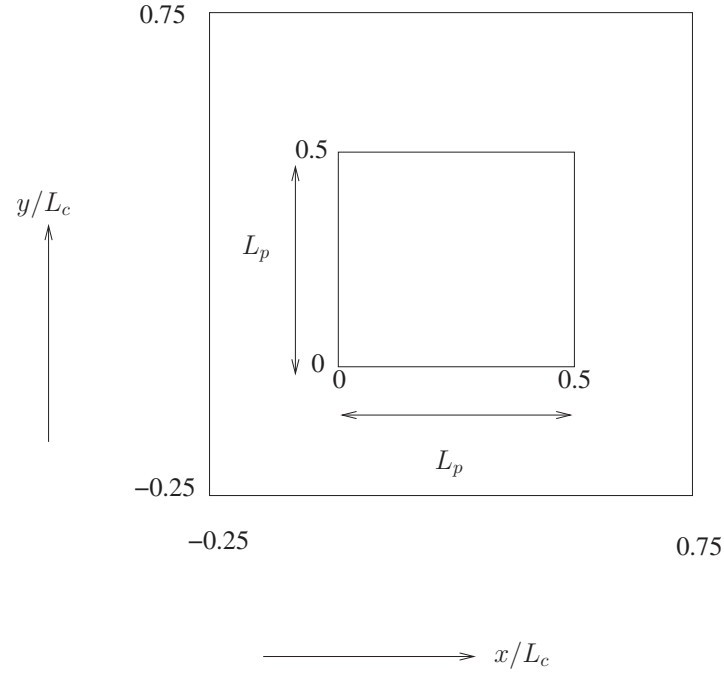

FIG. 2. The post cross-section, $L_{p} \times L_{p}$, and the cell crosssection, $L_{c} \times L_{c}$, where $L_{c}=2 L_{p}$.

about the topological classification, energy estimates, and regularity of equilibrium configurations in previous work $[9,10]$. In this paper, we apply similar methods to a complex nonconvex polyhedral geometry-the PABN geometry.

The paper is organized as follows. In Sec. II, we describe our simplified model of the PABN device, based on the Oseen-Frank continuum model. In Sec. III, we identify four distinct (but simple) topologies in this model geometry. We construct trial configurations with these topologies in Sec. IV. These trial configurations are used as initial conditions for a numerical solver of the equilibrium Euler-Lagrange equations (4). We obtain numerical solutions for each topological class. The energies and morphologies of these solutions are then correlated to the observed physical phenomena. The numerical results and experimental observations are in qualitative agreement.

\section{MODEL}

We work with a simplified model version of the PABN geometry, neglecting factors such as post tilt and replacing the rounded edges and corners in Fig. 1 by sharp features. Further, we only consider configurations which are periodic in the array so that it suffices to look at what happens around a single post. (This is a realistic assumption since a typical pixel in the PABN device consists of thousands of these posts.)

Our model geometry is displayed in Figs. 2 and $3-\mathrm{a}$ single rectangular post of fixed square cross section $\left(L_{p}\right.$ $\left.\times L_{p}\right)$ and variable height, $h$, inside a cell of fixed dimensions, $L_{c} \times L_{c} \times H$. The post and cell cross-sectional parameters, $L_{p}$ and $L_{c}$, respectively, are chosen so that $L_{c}=2 L_{p}$, whereas the cell height $H$ is fixed to be $H=3 L_{c}$. These choices roughly agree with the actual device parameters.

We model the liquid crystal configuration, outside the rectangular post, by a unit-vector field $\mathbf{n}(\mathbf{r})$. We impose periodic conditions on the cell boundaries so that

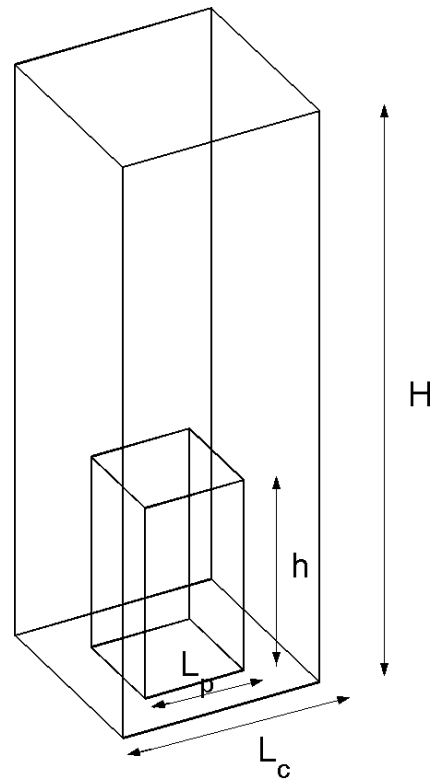

FIG. 3. The model geometry with $L_{c}=1, L_{p}=0.5$, and $H=3$. When translated to physical units, these correspond to $L_{c}=1 \mu \mathrm{m}$, $L_{p}=500 \mathrm{~nm}$, and $H=3 \mu \mathrm{m}$, respectively.

$$
\mathbf{n}(x, y, z)=\mathbf{n}\left(x+L_{c}, y, z\right)=\mathbf{n}\left(x, y+L_{c}, z\right), \quad \text { etc } .
$$

The constraint (1) allows us to extend our results to a periodic array of posts. Further, $\mathbf{n}$ is taken to satisfy tangent boundary conditions on the bottom substrate and post surfaces and normal conditions on the top substrate, consistent with the boundary conditions in the device.

Working within the Oseen-Frank continuum model, the liquid crystal energy is given by

$$
E[\mathbf{n}]=\int_{V} w(\mathbf{n}, \nabla \mathbf{n}) d V
$$

where

$$
\begin{aligned}
w(\mathbf{n}, \boldsymbol{\nabla} \mathbf{n})= & K_{1}(\boldsymbol{\nabla} \cdot \mathbf{n})^{2}+K_{2}(\mathbf{n} \cdot \boldsymbol{\nabla} \times \mathbf{n})^{2}+K_{3}(\mathbf{n} \times \boldsymbol{\nabla} \times \mathbf{n})^{2} \\
& +\left(K_{2}+K_{4}\right)\left[\operatorname{tr}(\boldsymbol{\nabla n})^{2}-(\boldsymbol{\nabla} \cdot \mathbf{n})^{2}\right]
\end{aligned}
$$

and the $K_{j}$ are material-dependent elastic constants [1]. The stable configurations then correspond to local minimizers of (2), subject to the imposed boundary conditions.

\section{TOPOLOGY}

Our aim in this paper is to identify and analyze topologically distinct stable configurations in the PABN cell (i.e., configurations that cannot be continuously deformed into each other). In this section, we first study the twodimensional zenithally bistable nematic (ZBN) cell as an illustrative example $[11,12]$. The $\mathrm{ZBN}$ cell has a comparatively simple two-dimensional geometry where the topology is characterized by a single quantity-the planar winding number [13]. Here, we look at the experimentally observed 
states and identify their distinct topologies. Then, we study the three-dimensional PABN cell, where the geometry is far more complex and the topology richer. We identify four different topological classes in this geometry. These classes generate tilted and comparatively planar profiles around the rectangular post and, hence, serve as good candidates for the topologies of the experimentally observed tilted and planar states.

\section{A. ZBN cell}

The ZBN cell, like most liquid crystal cells, consists of a liquid crystal layer sandwiched between two substrates. The bottom substrate is planar whereas the upper substrate is a monograting. Both substrates are treated to be homeotropic so that $\mathbf{n}$ is constrained to be normal to these surfaces.

The ZBN cell supports two stable, optically contrasting configurations - the high-tilt almost vertical state which is dark when viewed under crossed polarizers and the low-tilt state, supporting greater bulk distortion, that is bright under crossed polarizers [11,12] (tilt is measured with respect to the horizontal direction). The cell cross section (with a wedge-shaped upper substrate) and the director profiles for the two observed states are shown in Figs. 4(a) and 4(b).

The topology of these states is characterized by the rotation of $\mathbf{n}$ along a path connecting the two sides of the grating, as shown in Fig. 4. This rotation can be quantified in terms of the winding number - a topological invariant such that two unit-vector fields $\mathbf{n}_{1}$ and $\mathbf{n}_{2}$ in the ZBN geometry are topologically equivalent if and only if they have the same winding number. It clearly follows from Fig. 4that the high-tilt state has the minimum allowed rotation consistent with the boundary conditions whereas the low-tilt state exhibits greater intermediate rotation. In fact, the net rotations for the two states differ by $\pi$ or a half-winding number [13]. This then implies that the two states have different winding numbers and are thus topologically distinct, leading to their longterm stabilities.

It should be noted that $\mathbf{n}$ can, in principle, rotate by arbitrarily large amounts around the relief grating (between the fixed orientations at the sides) but such states have much higher energies and are unlikely to be observed in practice.

\section{B. PABN cell}

Next, we consider the three-dimensional model PABN cell, in Fig. 3. Here, the topology cannot be merely characterized by the planar winding number, as in the ZBN case. In fact, there are at least three separate topological invariants on

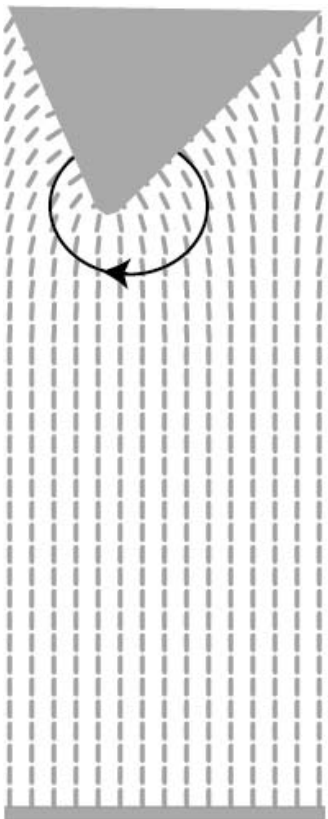

(a)

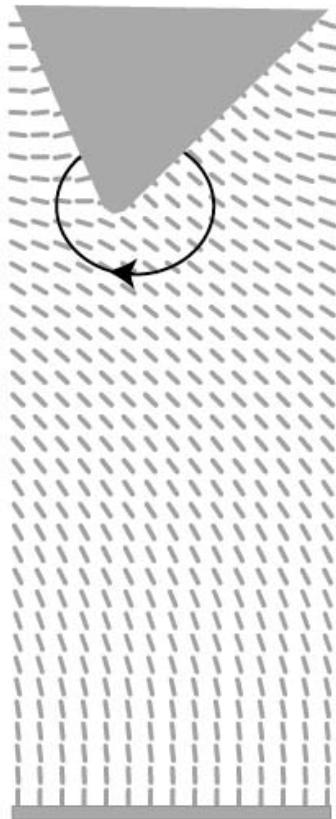

(b)
FIG. 4. (a) The high-tilt state. (b) The low-tilt state.

the post edges, post faces, and around the post vertices. In this section, we do not give a detailed description of the topological classification but identify four distinct, lowenergy topologies. A systematic account is given in $[6,7]$.

We first recall that the tangent boundary conditions on the bottom substrate and the post surfaces imply that on these surfaces, $\mathbf{n}$ takes values tangent to these surfaces. Therefore, on the post edges, $\mathbf{n}$ is parallel to the edges and can take one of two possible values. The value of $\mathbf{n}$, on a post edge, is defined to be the corresponding edge orientation [6]. $\mathbf{n}$ is necessarily discontinuous at the vertices, where three or more edges meet. For simplicity, we only consider configurations which are continuous everywhere away from the sharp post vertices.

We first look at the top face of the post and the corresponding four horizontal edges. Up to symmetry and the sign of $\mathbf{n}$, there are three distinct choices of the horizontal edge orientations on this face, as shown in Fig. 5. The last case necessarily creates a planar defect on this face and is, therefore, excluded (the constraint of interior continuity disallows certain choices of the edge orientations, for details see [6]). Of the remaining two cases, we choose the first one since it is more symmetric and is, therefore, expected to have lower energy. Once the horizontal orientations on the top face are fixed as in Fig. 5(a), the horizontal orientations on the corre-

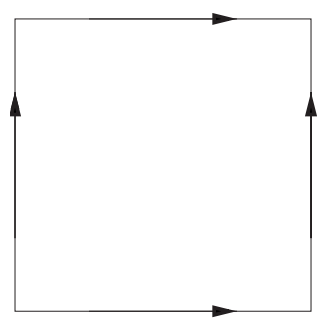

(a)

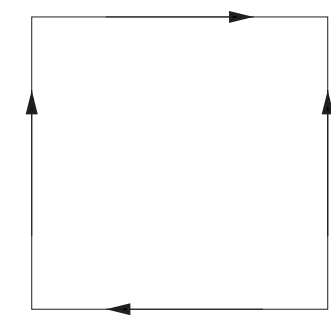

(b)

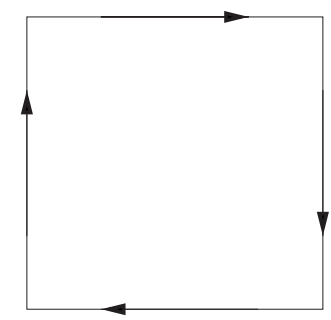

(c)
FIG. 5. (a) The most symmetric choice. (b) Admissible choice of lower symmetry. (c) Necessarily contains a planar defect. 


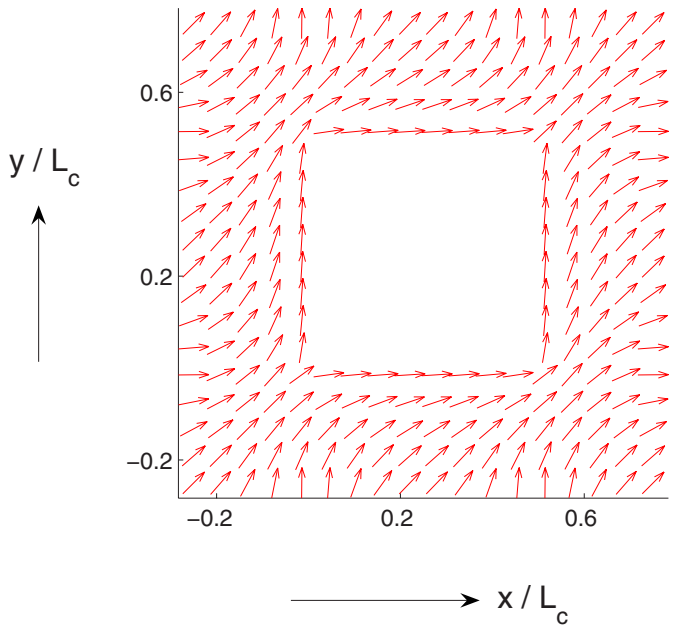

(a)

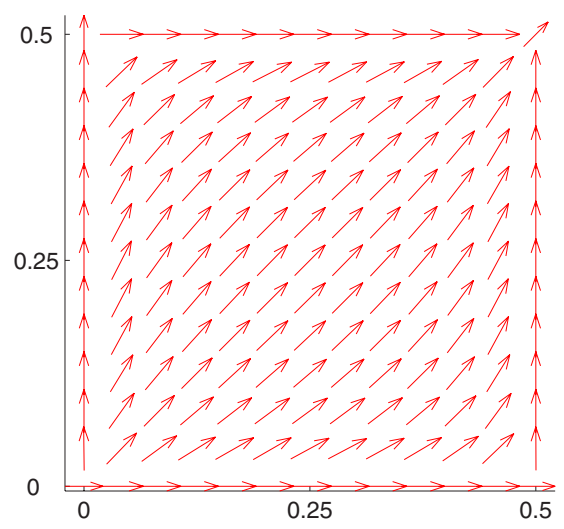

(b)

FIG. 6. (Color online) (a) The profile on the bottom substrate. (b) The profile on the top face of the post.

sponding bottom edges are taken to coincide with those on the top. This then completely determines the edge orientations on the eight horizontal post edges in our model, which are kept fixed for simplicity. (See Fig. 6.)

We then consider the edge orientations on the four vertical post edges. We recall that tangent boundary conditions imply that $\mathbf{n}$ is either oriented upwards or downwards on these edges. Labelling the four edges by $i=\{1,2,3,4\}$ as in Fig. 7 and the corresponding edge orientation by $\mathbf{e}_{i}$, there are four distinct cases up to symmetry and the sign of $\mathbf{n}$. These cases are enumerated in Table I. The first case, $T$, corresponds to $\mathbf{n}$ being oriented upwards on all four edges. For the second case, $P_{1}, \mathbf{n}$ is oriented upwards on three vertical edges and downwards on the remaining fourth edge whereas for the cases $P_{2}$ and $P_{3}, \mathbf{n}$ is oriented upwards on two edges and downwards on the remaining two. $P_{2}$ and $P_{3}$ are distinguished by the fact that $\mathbf{e}_{i}$ changes sign on a pair of opposite faces in $P_{2}$ whereas $\mathbf{e}_{i}$ changes sign on all four vertical faces in $P_{3}$.

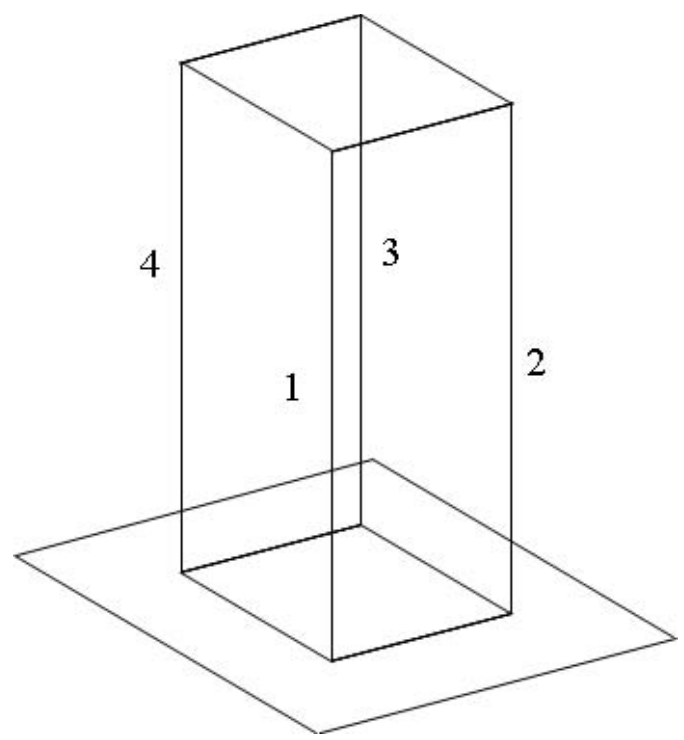

FIG. 7. The four vertical edges.
In addition to the edge orientations, the topology of $\mathbf{n}$ is also determined by its behavior on the post faces and around the post vertices. We take an arbitrary path connecting a pair of adjacent post edges, on a post face. The tangent boundary conditions imply that as we move along this path, $\mathbf{n}$ rotates in the plane of the corresponding face. We only consider topologies whereby $\mathbf{n}$ undergoes minimal rotation between pairs of adjacent post edges (which is $\pm \frac{\pi}{2}$ or a quarter turn). In the terminology of [6], we say that these topologies have zero kink numbers.

The fixed horizontal edge orientations and the constraint of zero kink numbers allows us to qualitatively predict the behavior of $\mathbf{n}$ on the bottom substrate and the top of the post, as shown in Figs. 6(a) and 6(b). On the bottom substrate, our choice of the horizontal orientations implies that $\mathbf{n}$ splits at one of the vertices (the bottom left-hand vertex), follows the post edges and then rejoins at the top right-hand vertex, aligning along the square cross-sectional diagonal. The net rotation between any two adjacent edges is just $\pm \frac{\pi}{2}$, as is evident from Fig. 6(a). Similarly, on the top of the post [refer to Fig. 6(b)], we have a fractional source defect at one of the vertices (the bottom left-hand vertex), accompanied by a sink defect at the diagonally opposite (top right-hand) vertex and any continuous $\mathbf{n}$ aligns along the corresponding diagonal.

The sharp post vertices can be treated as fractional point defects in our model; the corresponding strength is measured in terms of the neighboring distortion around the vertex. We only consider topologies where $\mathbf{n}$ has minimal distortion around post vertices or equivalently, these vertices are fractional defects of minimal degree (the solid angle subtended

TABLE I. The four sets of vertical orientations.

\begin{tabular}{lcccc}
\hline \hline & $\mathbf{e}_{1}$ & $\mathbf{e}_{2}$ & $\mathbf{e}_{3}$ & $\mathbf{e}_{4}$ \\
\hline$T$ & $\hat{\mathbf{z}}$ & $\hat{\mathbf{z}}$ & $\hat{\mathbf{z}}$ & $\hat{\mathbf{z}}$ \\
$P_{1}$ & $\hat{\mathbf{z}}$ & $\hat{\mathbf{z}}$ & $-\hat{\mathbf{z}}$ & $\hat{\mathbf{z}}$ \\
$P_{2}$ & $\hat{\mathbf{z}}$ & $-\hat{\mathbf{z}}$ & $-\hat{\mathbf{z}}$ & $\hat{\mathbf{z}}$ \\
$P_{3}$ & $\hat{\mathbf{z}}$ & $-\hat{\mathbf{z}}$ & $\hat{\mathbf{z}}$ & $-\hat{\mathbf{z}}$ \\
\hline \hline
\end{tabular}




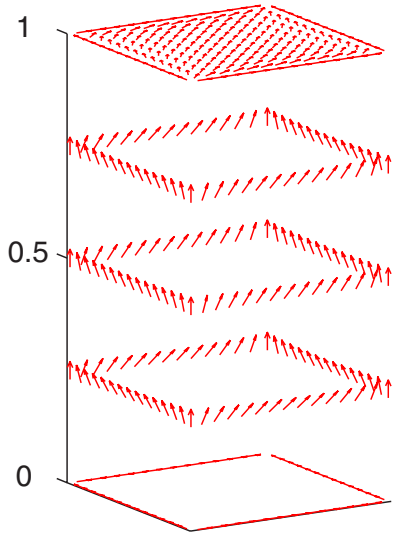

(a)

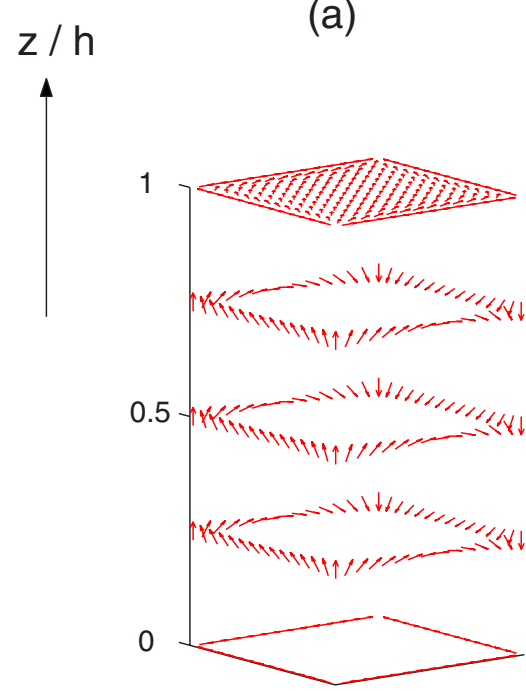

(c)

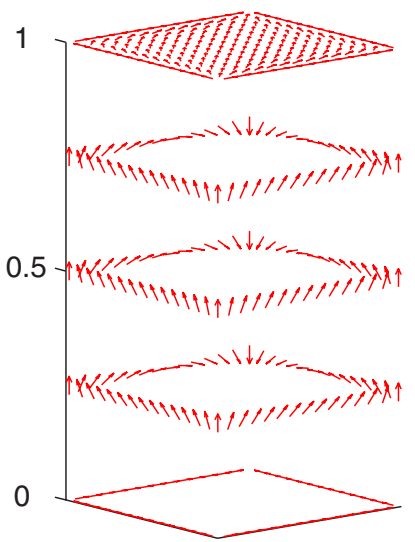

(b)

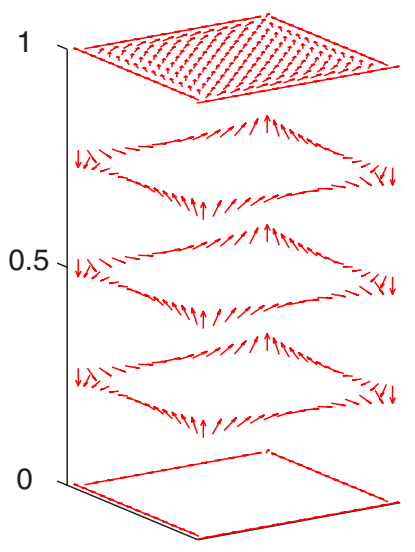

(d)

FIG. 8. (Color online) (a) The $T$ profile. (b) The $P_{1}$ profile. (c) The $P_{2}$ profile. (d) The $P_{3}$ profile. The scaled vertical coordinate in each plot is $z / h$, where $h$ is the post height and $0 \leqslant z / h \leqslant 1$.

by $\mathbf{n}$ as it varies around a post vertex is just $\left.\pm \frac{\pi}{2}\right)$. In the terminology of [6], these topologies have minimal trapped areas.

This then defines four distinct topologies, $\left\{T, P_{1}, P_{2}, P_{3}\right\}$, all of which have fixed horizontal orientations as in Fig. 5(a), zero kink numbers and minimal trapped areas but are distinguished by their vertical orientations given in Table I. The topologies are labelled by their vertical orientations for simplicity, since the vertical orientations are the only topological parameters in our model.

\section{NUMERICAL MODELLING}

We now investigate the existence of equilibrium stable configurations with the four topologies cited in Sec. III. To this end, we use the finite-element method to numerically solve the Euler-Lagrange equations associated with the Oseen-Frank energy (2) [14],

$$
\frac{\partial}{\partial \mathbf{n}_{i}} w(\mathbf{n}, \boldsymbol{\nabla n})=\frac{\partial}{\partial r_{j}}\left(\frac{\partial}{\partial \mathbf{n}_{i, j}} w(\mathbf{n}, \boldsymbol{\nabla n})\right),
$$

where $w(\mathbf{n}, \nabla \mathbf{n})$, the energy density, has been defined in (3), $\mathbf{n}_{i}$ is the $i$ th component of the unit-vector field, and $\mathbf{n}_{i, j}$ is the $j$ th partial derivative of $\mathbf{n}_{i}$. The numerical modeling is carried out in Femlab - a commercial partial differential equation solver based on the finite-element method [15].

We first construct trial configurations for each of the four topologies. Here, it is simpler to specify an unnormalized vector field $\mathbf{N}=\left(N_{x}, N_{y}, N_{z}\right)$. The unit-vector field $\mathbf{n}$ is then given by

$$
\mathbf{n}=\frac{\mathbf{N}}{|\mathbf{N}|} .
$$

The fixed horizontal orientations allow us to have a single prescription for $N_{x}$ and $N_{y}$ in the four cases, 

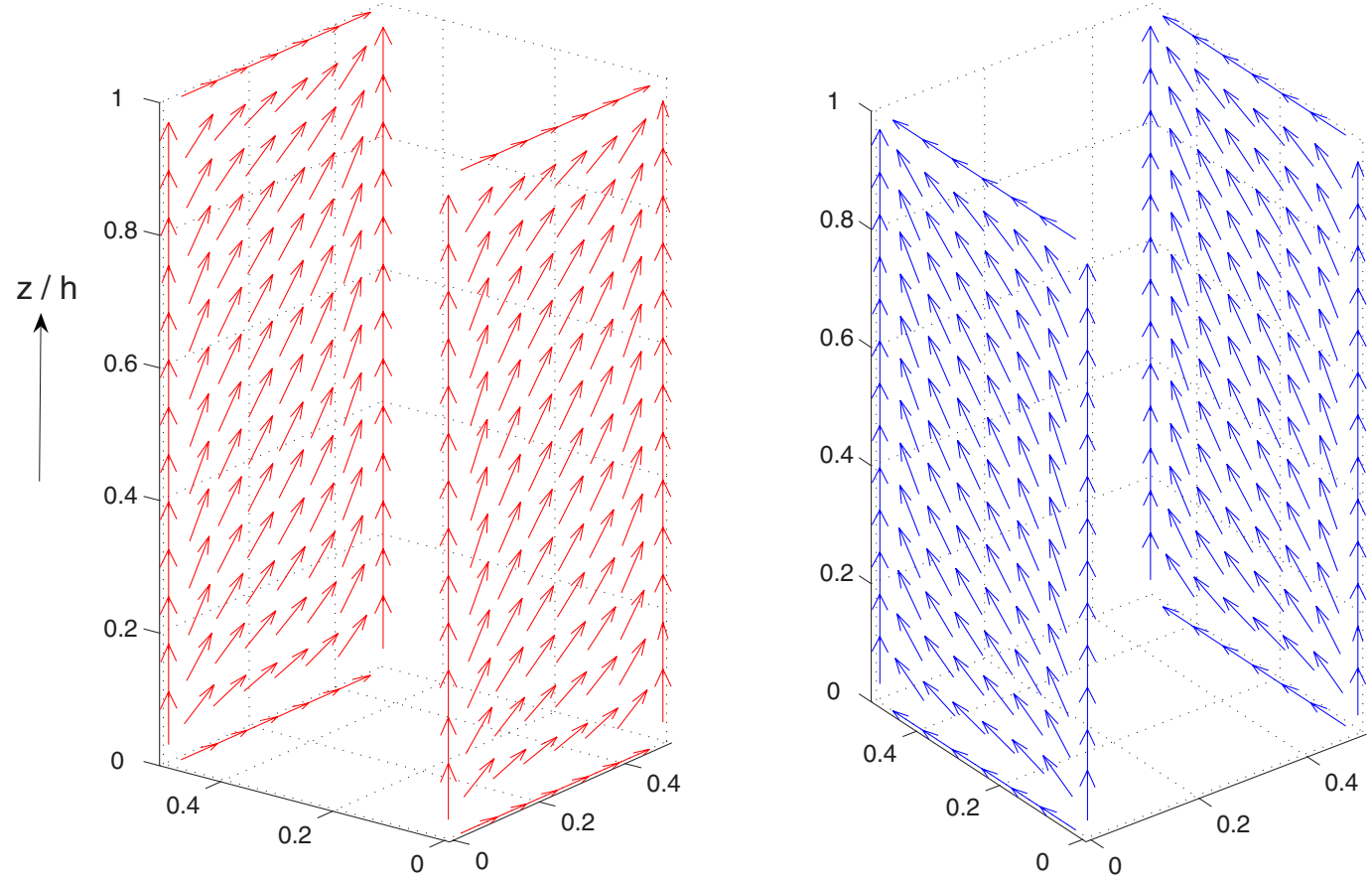

FIG. 9. (Color online) The $T$ profile on the faces, up to the height of the post $0 \leqslant z \leqslant h$, where $z$ is in $\mu$ m. The vertical coordinate is the dimensionless quantity- $z / h$ where $0 \leqslant z / h \leqslant 1$.
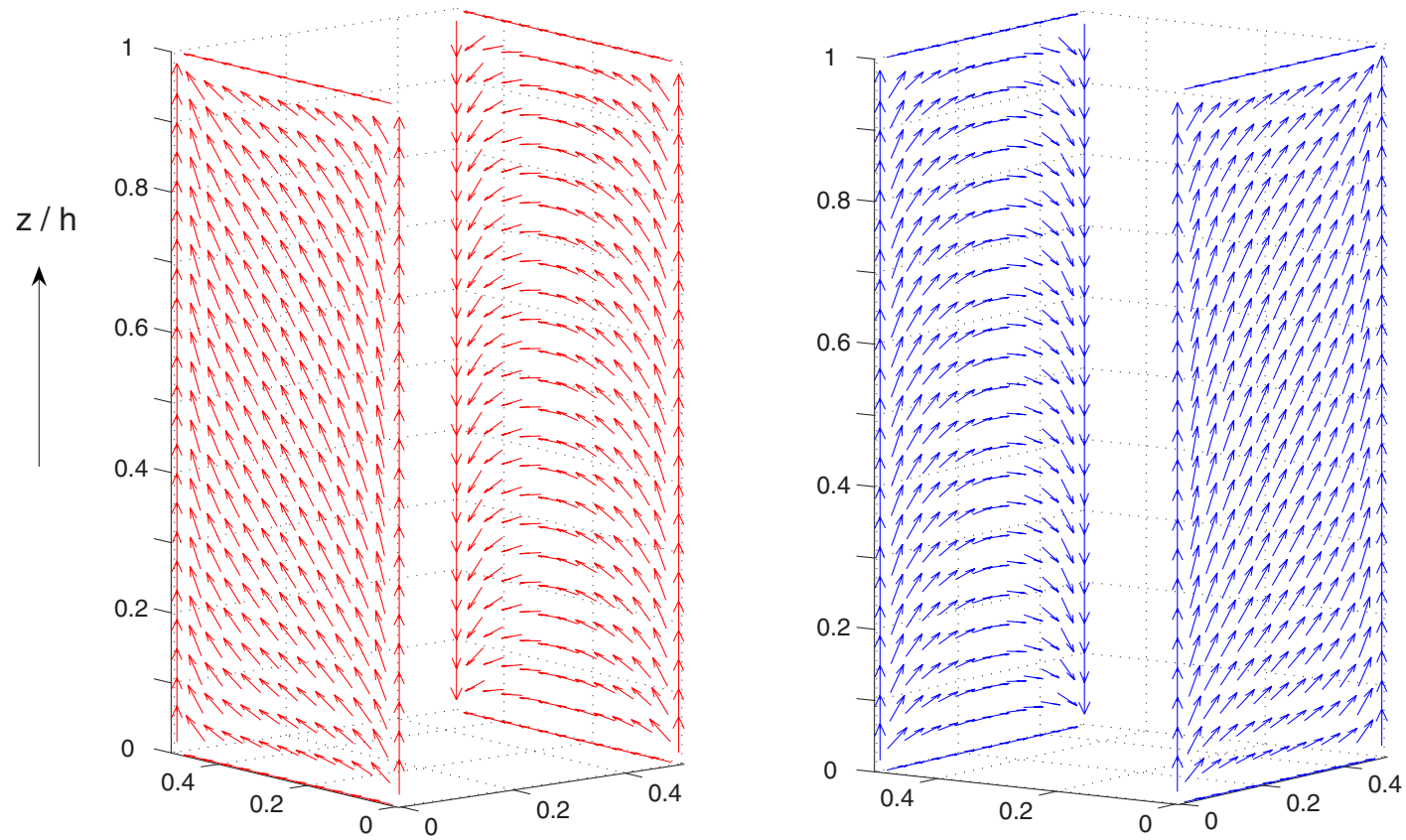

FIG. 10. (Color online) The $P_{1}$ profile on the post faces. 

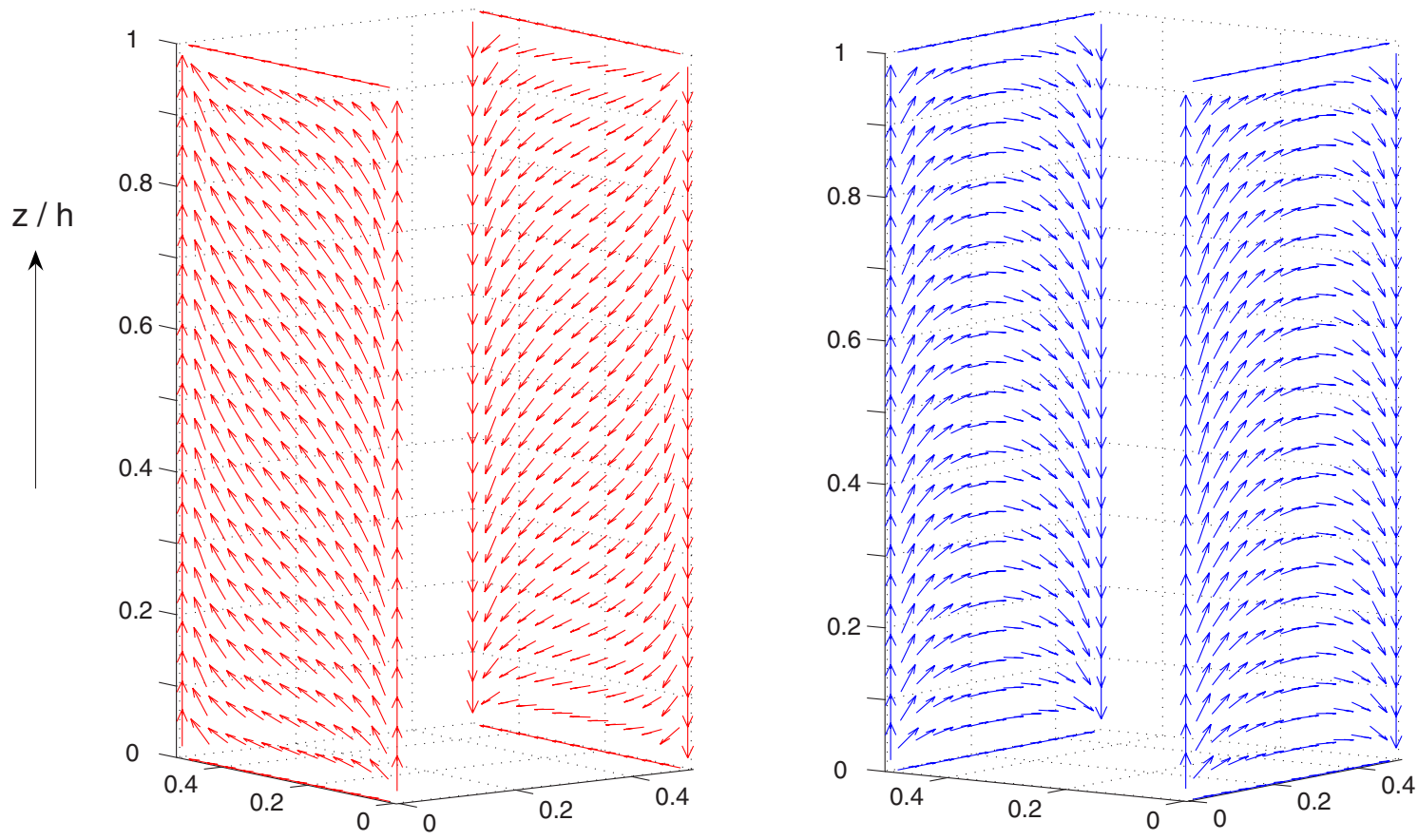

FIG. 11. (Color online) The $P_{2}$ profile on the post faces.
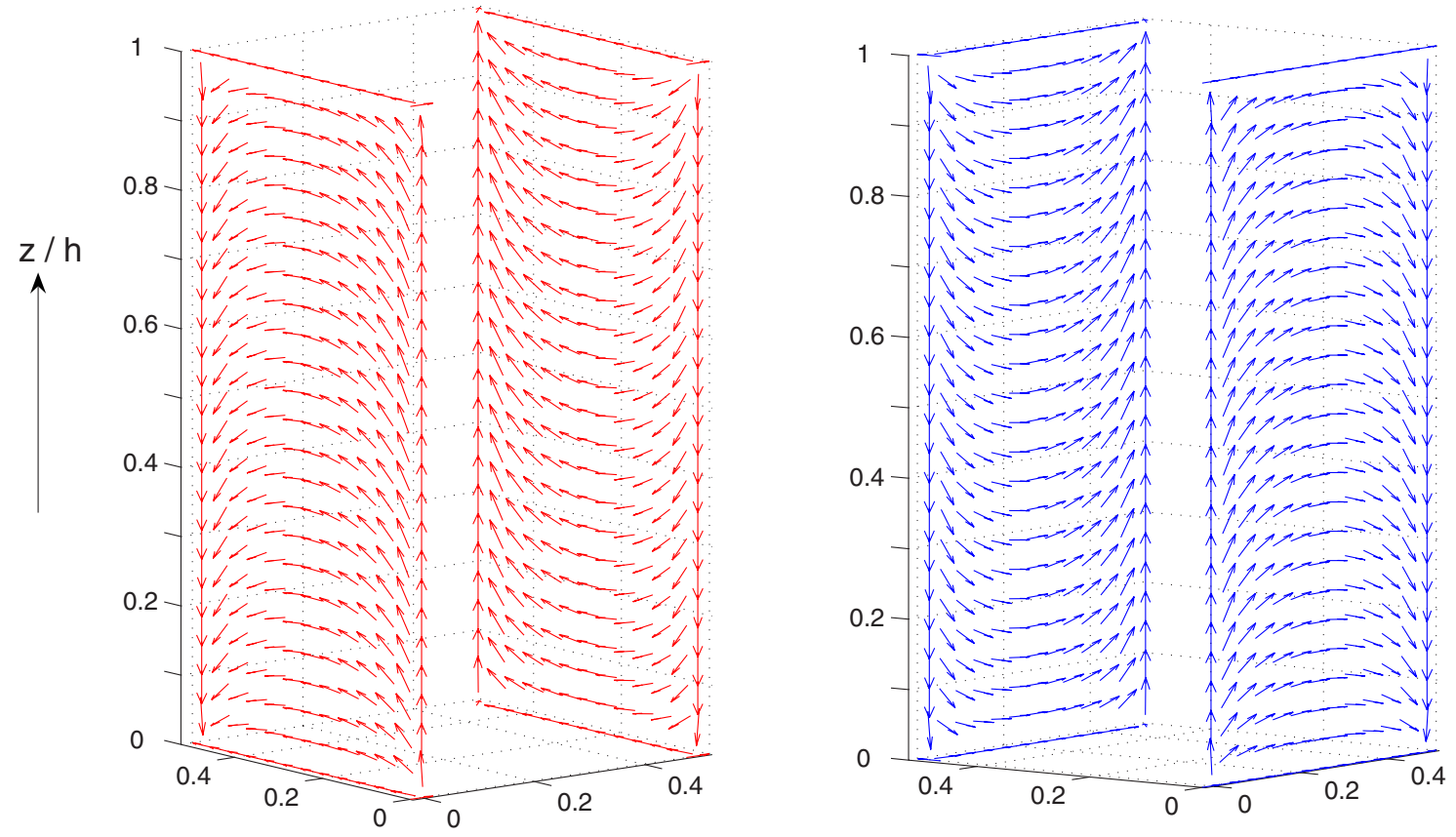

FIG. 12. (Color online) The $P_{3}$ profile on the post faces. 


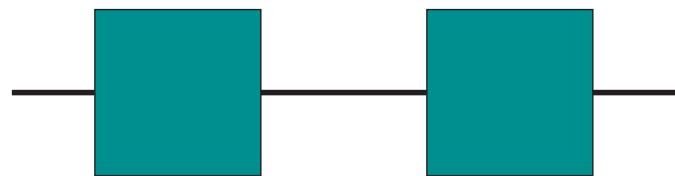

FIG. 13. (Color online) A periodically extended domain containing two posts. This is the bottom cross section, with the filled regions corresponding to the post bases. The bold line indicates the cross section $y=L_{c} / 4$, which is plotted in Figs. 14-17.

$$
\begin{aligned}
& N_{x}=\left[\sin \left(\frac{\pi x}{L_{p}}\right)\right]^{2}\left(\frac{H-z}{H}\right), \\
& N_{y}=\left[\sin \left(\frac{\pi y}{L_{p}}\right)\right]^{2}\left(\frac{H-z}{H}\right),
\end{aligned}
$$

where $L_{p}$ is the post cross-sectional parameter and $H$ is the cell height.

For $0 \leqslant z \leqslant h$, up to the height of the post, $N_{z}$ clearly has different forms to account for the different sets of vertical orientations,

$$
N_{z}= \begin{cases}z(h-z), & T, \\ {[z(h-z)]\left[1+\cos \left(\frac{\pi x}{L_{p}}\right)+\cos \left(\frac{\pi y}{L_{p}}\right)\right],} & P_{1}, \\ {[z(h-z)] \cos \left(\frac{\pi x}{L_{p}}\right),} & P_{2}, \\ {[z(h-z)] \cos \left(\frac{\pi x}{L_{p}}\right) \cos \left(\frac{\pi y}{L_{p}}\right),} & P_{3} .\end{cases}
$$

For $h \leqslant z \leqslant H$ (from the top of the post to the top substrate), $N_{z}$ is given by

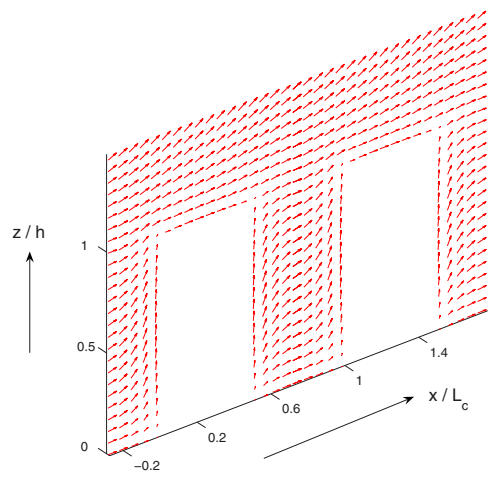

FIG. 14. (Color online) This plot is the projection of the $T$ profile onto the plane $y=L_{c} / 4$, i.e., only the $x$ and $z$ components are shown here. The dots, in particular, correspond to points where the solution is oriented in the $y$ direction. The horizontal coordinate is the dimensionless quantity $x / L_{c}$ and the vertical coordinate is $z / h$.

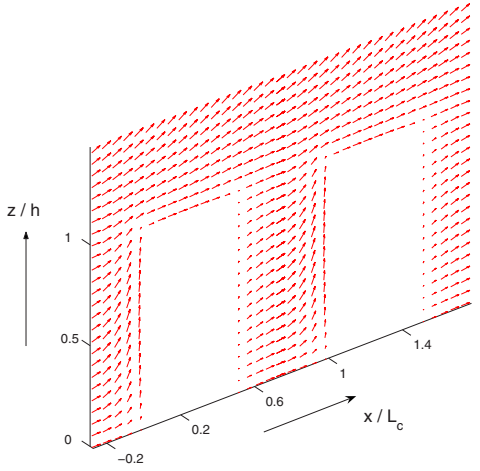

FIG. 15. (Color online) The projection of the $P_{1}$ profile onto the plane $y=L_{c} / 4$.

$$
N_{z}=\frac{z-h}{H-h}
$$

for all topologies. (Note that for $L_{c}=2 L_{p}$ and $\frac{-1}{4} \leqslant \frac{x}{L_{c}}, \frac{y}{L_{c}}$ $\leqslant \frac{3}{4}, 0 \leqslant z \leqslant H$, the only points where $\mathbf{N}$ vanishes are given by the post vertices, which are necessarily singularities because of the tangent boundary conditions.)

Given Eqs. (6)-(8), one can easily check that the representative (5) satisfies the boundary conditions with the correct topology and is continuous away from the post vertices. We use these trial configurations as initial conditions for the numerical solver. We numerically solve the Euler-Lagrange equations on a variable mesh that allows for greater resolution near the sharp post features. The numerical solver respects the boundary conditions and topology so that the numerical solutions have the same topology as the initial condition.

We show the solution profiles with $h=L_{c}$ in Figs. 8-12 and 14-17. We plot the profile around the rectangular post for $\left\{T, P_{1}, P_{2}, P_{3}\right\}$ in Figs. 8(a)-8(d). It is evident that the profiles are continuous and regular around the post with no point or line singularities. In particular, there are no defects along the leading and trailing edges, as suggested by previ-

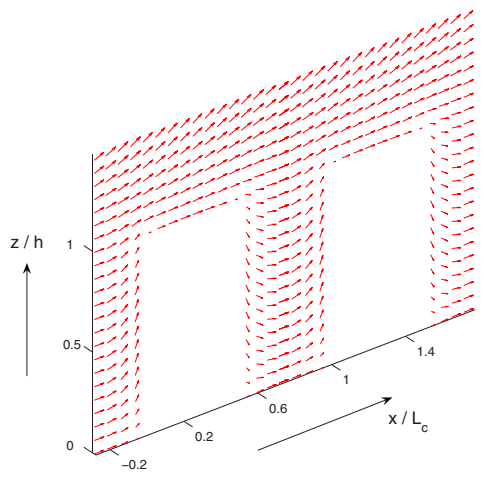

FIG. 16. (Color online) The projection of the $P_{2}$ profile onto $y$ $=L_{c} / 4$. 


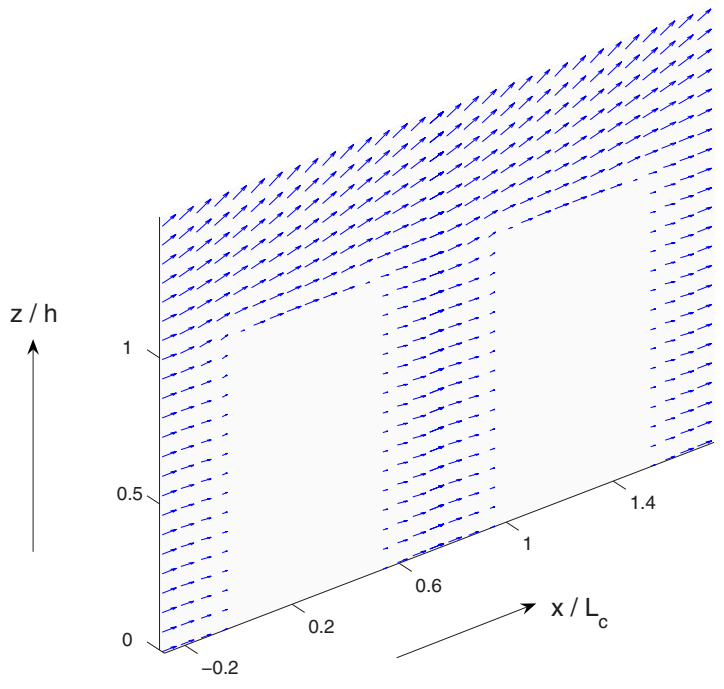

FIG. 17. (Color online) The projection of the $P_{3}$ profile onto $y$ $=L_{c} / 4$. Note that we only plot up to height $z=3 h / 2$ because the profiles have similar structures in the remainder of the cell.

ous modeling [4]. In Figs. 9-12 we describe the four profiles on the post faces in greater detail. Figure 9 clearly shows that the $T$ profile tilts upwards at all points as we move across the post faces and aligns along the post diagonal. Figures 10-12 correspond to the $P_{1}, P_{2}$, and $P_{3}$ profiles, respectively. Here, we note that whenever the vertical orientation changes sign between a pair of successive vertical edges on a post face, there is necessarily an intermediate planar region. This planar region is typically a curve across the face, between the two edges in question. We can clearly see these planar regions on a pair of adjacent faces in the $P_{1}$ profile, on a pair of opposite faces in the $P_{2}$ profile and on all four vertical faces in the $P_{3}$ profile.

In Figs. 14-17, we plot the solutions on a periodically extended domain, comprising of a pair of posts, along the cross section $y=L_{c} / 4$ as shown in Fig. 13. The periodic boundary conditions imply that the profiles are simply repeated between neighboring posts. In particular, the solution topology is the same for every post. This is illustrated in Fig. 14, where the $T$ profile tilts upwards between a pair of neighboring posts, while interpolating between the fixed orientations on the successive vertical edges. Similarly, the planar regions observed in Figs. 10-12 extend between neighboring posts in Figs. 15-17, by virtue of the periodic boundary conditions. This is most pronounced for the $P_{3}$ profile, as might be expected on topological grounds. Above the post, for $h$ $\leqslant z \leqslant H$, all profiles tilt upwards and finally become homeotropic on the top substrate. These profiles, though discussed for a fixed post height $h=L_{c}$, are characteristic of the solution profiles for all $h$.

Next we look at how the solution energy varies with scaled post height, $h / L_{c}$, for two different sets of elastic constants in Figs. 18 and 19. The second set, with equal elastic constants, corresponds to the widely used one-constant approximation [1,14]. It is evident from Figs. 18 and 19 that the qualitative trends for the two sets of elastic constants are the same. However, the one-constant approximation in Fig. 19 is found to be computationally less demanding than the unequal constant case and allows for greater numerical resolution.

We first note that the $T$ solutions have the smallest energy whereas the $P_{3}$ solutions have the highest (there is a slight crossover between the $P_{2}$ and $P_{3}$ solutions in Fig. 18 but the free energy difference is negligible). This is consistent with experimental observations which show that the liquid crystal always relaxes into the high-tilt state, when cooled down from the isotropic state [4].

These energy trends can be anticipated and explained on topological grounds. As is evident from Figs. 9 and 14, the $T$ solution has minimal distortion consistent with the boundary conditions. The $P_{3}$ solution, on the other hand, has the most strongly distorted profile because of the planar regions on every vertical post face and between every pair of neighboring posts. These planar regions arise from the continuous interpolation between opposite vertical orientations; these vertical orientations, in turn, are part of the topological char-

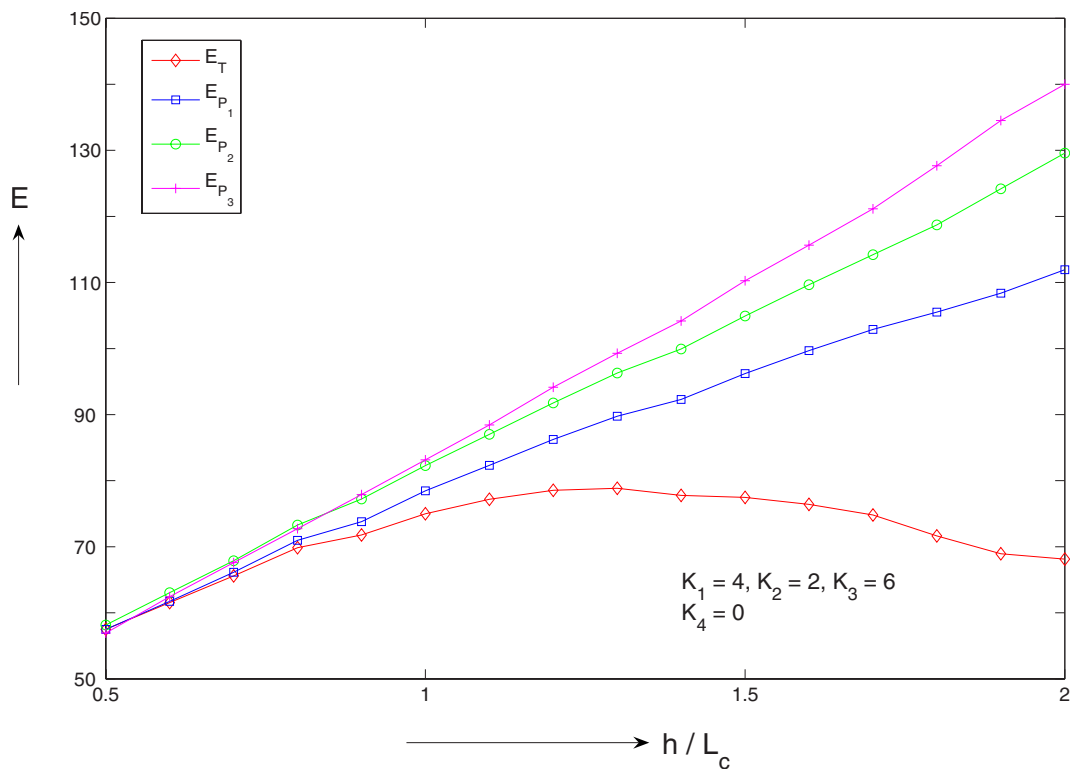

FIG. 18. (Color online) The solution energies $E$ in the OseenFrank case with $K_{1}=4, K_{2}=2$, and $K_{3}=6$ versus the scaled post height $h / L_{c}$. 


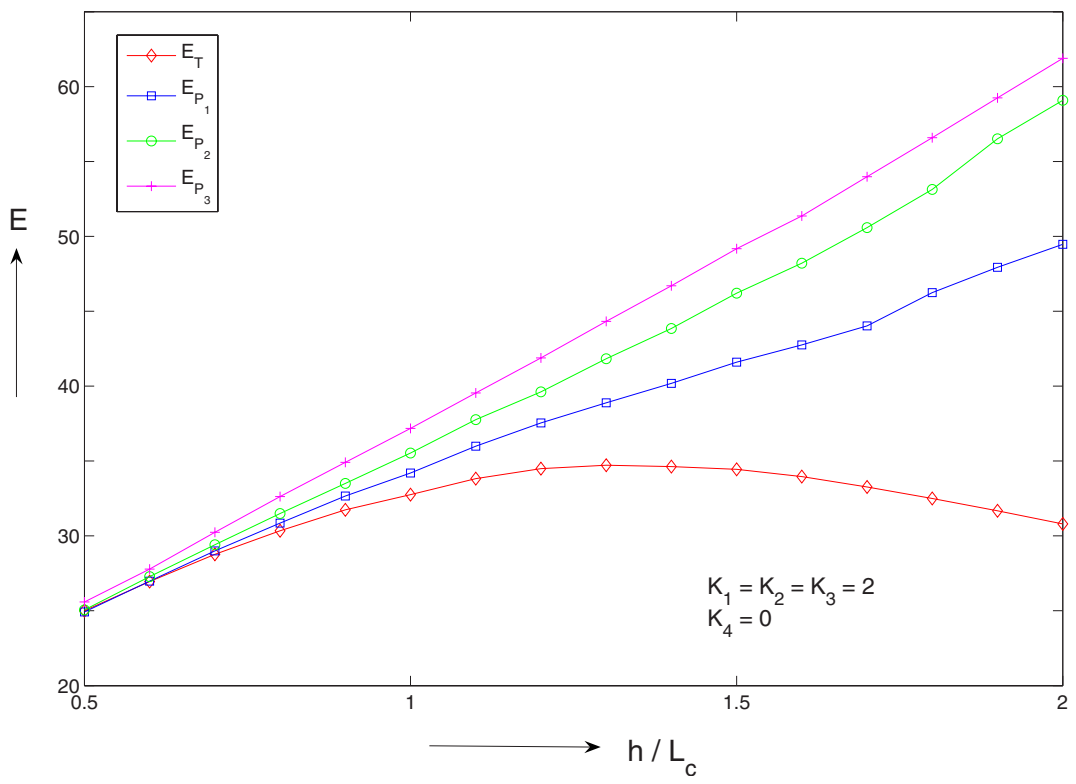

FIG. 19. (Color online) The solution energies $E$ in the oneconstant approximation with $K_{1}$ $=K_{2}=K_{3}=2$ versus the scaled post height $h / L_{c}$. acterization of these solution profiles. The minimal and maximal distortion, in the $T$ and $P_{3}$ solutions, respectively, then qualitatively explain the energy trends.

We look at the free energy differences in Fig. 20. Here, we plot two quantities:

$$
\epsilon_{1}=\frac{E_{P_{1}}-E_{T}}{E_{T}}
$$

and

$$
\epsilon_{3}=\frac{E_{P_{3}}-E_{T}}{E_{T}}
$$

for the elastic constants in Fig. 18. $E_{P_{1}}$ is typically the smallest of $\left\{E_{P_{1}}, E_{P_{2}}, E_{P_{3}}\right\}$ and $E_{P_{3}}$ is the largest. It is evident from Fig. 20 that both $\epsilon_{1}$ and $\epsilon_{3}$ are small and slowly increasing for scaled post heights $h / L_{c}$ in the interval $h / L_{c} \in[0.5,0.9]$.
We refer to this interval, $h \in\left[0.5 \times L_{c}, 0.9 \times L_{c}\right]$, as the shallow region in the remaining discussion. For post heights $h$ $\geqslant L_{c}$, the gradient of both curves increases appreciably so that both $\epsilon_{1}$ and $\epsilon_{3}$ grow sharply with post height. This change in behavior as a function of post height can be explained by Fig. 18. $E_{T}$, the energy of the tilted solution, typically reaches a shallow maximum and then decreases very slowly as the post height increases. On the other hand, both $E_{P_{1}}$ and $E_{P_{3}}$ grow linearly with post height. These differences in the energy trends manifest in $\epsilon_{1}$ and $\epsilon_{3}$, given by (9) and (10), leading to the features cited above.

To understand what these numerical calculations mean in the context of the actual device, we first note that the tilted and planar states are observed experimentally for post heights in the range 0.6 microns to 1.2 microns. This bistable region corresponds to $h \in\left[0.6 \times L_{c}, 1.2 \times L_{c}\right]$ in our model. Further, as the posts become taller, the lower energy

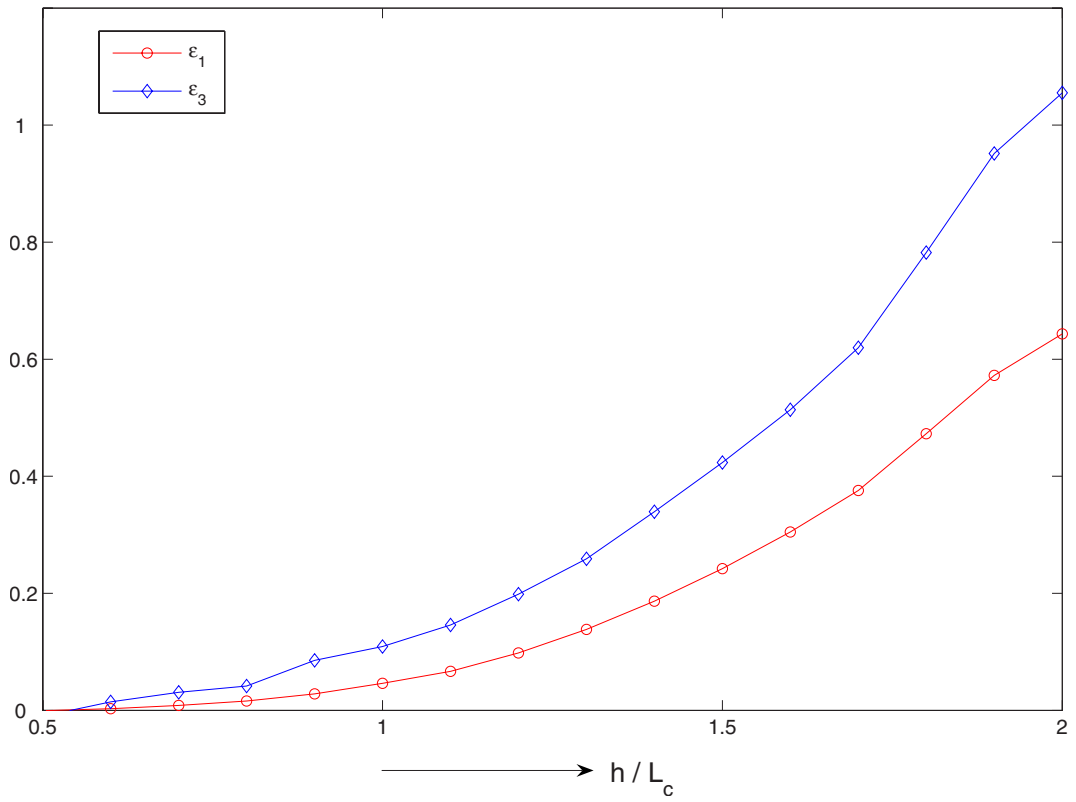

FIG. 20. (Color online) Computing $\epsilon_{1}$ and $\epsilon_{3}$ for the unequal constant case in Fig. 18 as a function of the scaled post height $h / L_{c}$. 
tilted state is more easily observed than the planar state and it becomes difficult to switch from the tilted state to the planar state [4]. Our modeling shows a shallow region (a region of slow growth) for $\frac{h}{L_{c}} \in[0.5,0.9]$, where both $\epsilon_{1}$ and $\epsilon_{3}$ are small and do not change significantly. This shallow region suggests that the topologically distinct $T$ and $P_{i}$ states, $i$ $=1,2,3$, have similar free energies for $\frac{h}{L_{c}} \in[0.5,0.9]$ and might be experimentally observed over this range. Our numerical range and the experimentally found bistable region are in qualitative agreement. Second, the fact that $\epsilon_{1}$ and $\epsilon_{3}$ grow quickly as a function of $\frac{h}{L_{c}}$ indicates that the $P_{i}$ states become energetically far more expensive than the $T$ states as the post height increases. This, in turn, qualitatively explains the experimental observations and switching characteristics for taller posts. A more detailed analysis of the liquid crystal energy would include surface effects and scalar order parameters $[1,14]$.

\section{CONCLUSION}

The methods and results presented in this paper illustrate how topology can be exploited in order to find different static liquid crystal configurations in prototype device geometries. For the specific case of the PABN geometry, we have identified four simple, low-energy topologies. This topological information is then used to construct suitable initial conditions for a finite-element numerical algorithm, yielding four distinct classes of numerical solutions. It would be difficult to numerically find these different solutions without the topological insight. Our modeling suggests that the tilted and planar states in the PABN geometry are topologically distinct, leading to a topological mechanism for the observed bistability. (For example, the $T$ topology defined in Sec. III is a good candidate for the topology of the tilted state whereas the $P_{i}$ topologies serve as good candidates for the planar state.) Second, these numerical solutions have comparable free energies over a certain range of post heights, indicating a limited bistability region that is again commensurate with experimental observations.

The methods outlined in this paper can be extended to more general polyhedral geometries. We can use topology to organize the space of admissible configurations, understand the structure of the stable configurations and their energetics. This will be investigated further in subsequent work.

\section{ACKNOWLEDGMENTS}

The preliminary $Q$-tensor modeling results of one of the authors (C.J.P.N.) and Dr. Nigel Mottram suggested a possible topological bistability mechanism in the PABN device, which we have numerically investigated and quantified in this paper. The authors thank them for sharing their modeling results. One of the authors (A.M.) would also like to thank the Royal Commission for the Exhibition of 1851 and EPSRC for financial support.
[1] P. De Gennes, The Physics of Liquid Crystals (Clarendon, Oxford, 1974).

[2] T. J. Sluckin, Contemp. Phys. 41, 37 (2000).

[3] G. Bryan-Brown, C. Brown, J. Jones, E. Wood, I. Sage, P. Brett, and J. Rudin, in SID Symposium Digest, Boston, edited by J. Morreale, 1997, p. 37.

[4] S. Kitson and A. Geisow, Appl. Phys. Lett. 80, 3635 (2004).

[5] A. J. Davidson and N. J. Mottram, Phys. Rev. E 65, 051710 (2002).

[6] J. M. Robbins and M. Zyskin, J. Phys. A 37, 10609 (2004).

[7] M. Zyskin (unpublished).

[8] C. Denniston and J. M. Yeomans, Phys. Rev. Lett. 87, 275505 (2001).

[9] A. Majumdar, J. M. Robbins, and M. Zyskin, J. Phys. A 37,
L573 (2004).

[10] A. Majumdar, J. M. Robbins, and M. Zyskin, Ann. Inst. Henri Poincare, Anal. Non Lineaire (to be published).

[11] C. V. Brown, M. J. Towler, V. C. Hui, and G. P. Bryan-Brown, Liq. Cryst. 27, 233 (2000).

[12] C. Newton and T. Spiller, Mol. Cryst. Liq. Cryst. Sci. Technol., Sect. A 372, 167 (2001).

[13] A. Majumdar, C. J. P. Newton, J. M. Robbins, and M. Zyskin, Hewlett Packard Technical Report, 2003 (http:// www.hpl.hp.com/techreports/2003/HPL-2003-66.html)

[14] I. W. Stewart, The Static and Dynamic Continuum Theory of Liquid Crystals (Taylor and Francis, London, New York, 2004).

[15] www.comsol.com 